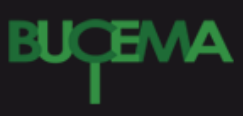

Bulletin du centre d'études médiévales d'Auxerre | BUCEMA

Hors-série $n^{\circ} 3 \mid 2010$

Présentation et mise en valeur des sites archéologiques religieux en milieu urbain

\title{
Réflexions sur les notions de conservation et de mise en valeur de vestiges archéologiques
}

\author{
Élise Faure-Boucharlat
}

\section{(2) OpenEdition}

Journals

Édition électronique

URL : https://journals.openedition.org/cem/11391

DOI : $10.4000 /$ cem. 11391

ISSN : 1954-3093

Éditeur

Centre d'études médiévales Saint-Germain d'Auxerre

Référence électronique

Élise Faure-Boucharlat, « Réflexions sur les notions de conservation et de mise en valeur de vestiges archéologiques ", Bulletin du centre d'études médiévales d'Auxerre | BUCEMA [En ligne], Hors-série n 3 | 2010, mis en ligne le 15 mars 2010, consulté le 03 mars 2023. URL : http://journals.openedition.org/ cem/11391 ; DOI : https://doi.org/10.4000/cem.11391

Ce document a été généré automatiquement le 3 mars 2023.

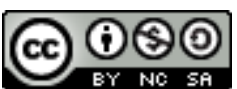

Creative Commons - Attribution - Pas d'Utilisation Commerciale - Partage dans les Mêmes Conditions 4.0 International - CC BY-NC-SA 4.0

https://creativecommons.org/licenses/by-nc-sa/4.0/ 


\title{
Réflexions sur les notions de conservation et de mise en valeur de vestiges archéologiques
}

\author{
Élise Faure-Boucharlat
}

1 Avant de conclure la table ronde de Luxeuil (25-26 avril 2008) consacrée à « $L a$ présentation et à la mise en valeur des sites archéologiques religieux en milieu urbain ", tout en gardant à l'esprit les cas d'école présentés au cours de ces deux journées,je propose de nous arrêter quelques instants sur les pratiques qui ont, en France, au fil du temps, présidé à la préservation d'un échantillon plus ou moins représentatif du patrimoine archéologique et de réfléchir aux processus scientifiques, culturels, économiques, voire politiques, qui ont conditionné les choix de leur conservation et de leur restitution au public.

2 En France, la notion de "site», au sens de parc, jardin ou crypte archéologique, ne s'impose pas d'emblée. Certes, ces termes désignent l'aboutissement d'un processus patrimonial, mais ils recouvrent des situations et des réalités fort diverses. Concrètement, il n'existe pas en France de label « site archéologique ", à l'inverse de ce qui existe, par exemple, pour les musées et les collections qu'ils abritent (dispositif renforcé par loi de 2002 sur les musées de France). Cela implique qu'il n'y a pas de législation spécifique, ni pour la préservation, ni pour la gestion des vestiges archéologiques qui rejoignent le droit commun du patrimoine bâti, la protection au titre des monuments historiques n'étant pas une obligation.

3 À l'échelle du territoire, on a, évidemment, affaire à un échantillon de sites fort disparate, formé au fil des générations, au hasard des découvertes et des circonstances et sous les effets de motivations diverses. En définitive, le seul dénominateur commun est la référence à des manifestations du passé, en général à partir de vestiges d'architectures ou d'activités anciennes. Dans les faits, le "site archéologique », au sens de vestiges conservés et mis en valeur, repose avant tout sur le principe d'un espace délimité et privilégié, destiné à la visite de non spécialistes, et, si possible, à la pédagogie. Si il n'y a pas d'encadrement réglementaire spécifique, il y a cependant des 
pratiques administratives, culturelles, voire sociales, qui orientent les choix et déterminent la « création » des sites archéologiques.

\section{Les conditions de « création » des sites aménagés}

4 Le site archéologique et les vestiges qu'il contient, ou est susceptible de contenir, doivent répondre à un certain nombre d'attentes et d'exigences et, en quelque sorte, franchir les étapes qui les conduiront de l'invention, par la fouille et l'étude scientifique, à la restitution au public.

\section{L'étape de la sélection}

Le site doit être reconnu et désigné comme remarquable ou exceptionnel. Entrent en jeu de nombreux critères, qui appartiennent tant au domaine de l'objectivité qu'à celui de la subjectivité. Parmi ces critères, on peut citer :

- l'intérêt historique, esthétique ou documentaire ;

- une signification particulière, un poids symbolique, en référence à une période, un événement, un lieu ;

- un caractère d'exception ou, au contraire, de représentativité ;

- un état de conservation et de lisibilité satisfaisant ou remarquable ;

- mais aussi, les conditions de la découverte, la personnalité de l'inventeur, du fouilleur, l'action militante d'une association, voire le contexte politique...

\section{L'étape de la conservation}

Cette étape combine plusieurs actions :

- la conservation physique des vestiges par des mesures matérielles appropriées (de la simple consolidation à la mise hors d'eau et hors d'air) ;

- la protection juridique, si possible par des mesures foncières concernant la propriété du terrain et des vestiges et par toute une panoplie de mesures réglementaires (Code de l'urbanisme, Code du patrimoine). Ces actions reposent en général sur un consensus; elles font alors l'objet d'accords amiables. Elle peuvent aussi être imposées par les pouvoirs publics au nom de l'intérêt général et entraînent alors des procédures contentieuses plus ou moins lourdes et surtout très longues.

\section{L'étape de la valorisation}

7 Cette étape découle de la décision de doter le site des moyens de sa survie et de sa restitution au public. Le site est appelé à connaître une vocation singulière où l'accueil et l'encadrement du public, dans leurs aspects matériels, pédagogiques et économiques, appelleront l'adjonction de divers équipements et l'entrée en piste de différents partenaires.

8 La première étape (celle de la sélection, au sens de reconnaissance) se fonde en priorité sur le critère de la valeur historique et esthétique. Il est déterminant, mais reste provisoire, car sujet à remise en cause, selon le lieu, l'époque ou les circonstances. La deuxième étape (celle de la conservation, de la patrimonialisation) tend à rendre irréversibles les effets de la sélection. Elle met, à proprement parler, le site archéologique et les vestiges immobiliers à l'abri des risques ordinaires. La troisième étape (celle de la restitution, de l'élaboration d'un discours à l'adresse d'un large 
public) retient le critère de la valeur patrimoniale et culturelle, en vertu de laquelle tel témoin du passé est estimé bien commun et digne de transmission entre générations, mais ne peut ignorer les contraintes de la conservation matérielle et le poids de la suggestion économique.

\section{Rapide panorama des pratiques en France}

Sans rentrer dans des dénombrements, nomenclatures et classifications, on peut considérer que le "catalogue » des sites archéologiques mis en valeur en France est le reflet de l'histoire de l'archéologie, en tant que discipline, et de l'évolution de la notion de patrimoine. Il porte aussi témoignage des représentations de l'Histoire favorisées par les pouvoirs publics et assimilés, plus ou moins consciemment, par les initiateurs des projets de valorisation.

10 À la fin du XIX et au début du $\mathrm{Xx}^{\mathrm{e}}$ siècle, alors que l'archéologie se confond avec la Préhistoire, les premières réalisations de protection, par la conservation, concernent rien moins que l'origine de l'humanité. Elles s'attachent presque exclusivement aux monuments ou gisements préhistoriques (abris sous roche et grottes ornées, monuments mégalithiques isolés ou grands alignements). Sur l'ensemble du territoire, on cherche également à préserver les gisements éponymes des grandes cultures matérielles de la Préhistoire ancienne (Solutré, Saint-Acheul, Le Moustier...). Ces protections (on ne parle pas encore de mise en valeur) sont antérieures à la loi de 1913 sur les monuments historiques : seule l'expropriation au profit de l'État garantit alors la sauvegarde du patrimoine ancien, tandis que de puissantes sociétés savantes se substituent aux pouvoirs publics, souvent impuissants avant la promulgation des grandes lois patrimoniales.

11 Parallèlement, au milieu du $\mathrm{XIX}^{\mathrm{e}}$ siècle, l'action politique s'appuie sur les sites emblématiques de l'histoire de France pour célébrer les mythes fondateurs de la Nation: la Gaule avant la Conquête, la résistance à l'envahisseur, largement mis en scène grâce à l'implication directe de l'Empereur archéologue, Napoléon III, sur les grands oppida du Centre de la France (Alésia, Bibracte, Gergovie, etc.). Le Midi de la France n'est pas en reste, doté, quant à lui, d'une autre charge symbolique, celle de l'ouverture de la Gaule de l'âge du Fer aux prestigieuses civilisations méditerranéennes: lancement, par exemple, des programmes d'Ensérune, Olbia, Entremont, Glanum...

12 Cette première génération d'actions de protection et de conservation s'intéresse également aux témoins majeurs de l'Antiquité classique qui ponctuent l'ensemble du territoire national (théâtres et amphithéâtres, temples et forum, thermes et aqueducs...), dont la plupart est, d'ailleurs, incorporée à la liste Mérimée depuis 1830. Leur mise en scène, jusque dans le chœur des villes modernes, participe à la reconnaissance de la supériorité technique et culturelle de «la civilisation galloromaine ».

13 Dans les premières décennies du xxe siècle, après les dégagements des " antiquaires ", une tradition érudite, largement ouverte sur l'archéologie étrangère et, surtout, assez éloignée des connotations idéologiques mentionnées ci-dessus, fait ressurgir de nombreuses capitales de Cité, du nord au sud de la France. On doit à l'action de ces érudits, qui ouvrent la voie d'une archéologie scientifique, certains des parcs archéologiques les plus vastes, portions de villes ou de faubourgs. Les cas de Bavay, 
Vaison-la-Romaine ou Saint-Bertrand-de-Comminges sont bien représentatifs de cette génération de sites, largement dégagés au cours du XIX siècle, fouillés plus scientifiquement au $\mathrm{xx}^{\mathrm{e}}$, et dont l'étude, la conservation et la valorisation sont toujours en cours. Il est à souligner que la période gallo-romaine est la bénéficiaire quasi exclusive de ces grands chantiers.

Accompagnant l'émergence de l'archéologie préventive, une autre série d'aménagement de sites archéologiques voit le jour après 1960, grâce à une réglementation et à une administration plus performantes. Les services déconcentrés de l'État, mis en place dans toutes les régions françaises dans les années 1965-1975, cherchent à imposer des réponses à l'accélération des destructions du patrimoine par les grands projets d'aménagement urbain, tout en sachant pouvoir s'appuyer sur des associations et groupes de pression particulièrement actifs. On gravit un degré dans le processus de patrimonialisation des témoins matériels du passé : ce n'est plus le fait de la seule décision des spécialistes et des politiques, d'une élite en somme, c'est aussi le résultat d'une action citoyenne.

Dans ces actions, souvent conduites dans l'urgence, c'est la valeur historique qui prime et le souci de la sauvegarde immédiate en vue de la transmission aux générations futures. Il ne s'agit pas tant de mettre en exergue des vestiges remarquables ou esthétiques que de s'attacher à préserver un patrimoine en devenir. Dans ce foisonnement d'initiatives, qui manquèrent parfois de concertation, les stratégies de conservation et les contraintes économiques n'ont pas toujours été anticipées. Par défaut de coordination entre les acteurs et aussi de moyens, l'étape de la valorisation a été parfois différée au détriment de l'intégrité même des vestiges, mais aussi au détriment de l'image des archéologues. Il faudra la loi-programme sur le patrimoine, votée en 1989 pour une durée de cinq ans, pour lancer ou achever quelques grands chantiers de restauration et de mise en valeur.

Aujourd'hui, le rythme des programmes de mises en valeur se ralentit, tandis que l'on assiste à un certain " essoufflement » de grands sites classiques (de Carnac à Glanum, en passant par Bavay ou Vienne Saint-Romain-en-Gal) dont les conditions d'accueil et l'accompagnement pédagogique réclament de nouveaux investissements. De nombreux sites urbains (cryptes ou jardins archéologiques) accusent une certaine désaffection du public, souvent consécutive au désintérêt des propriétaires et gestionnaires. Par ailleurs, en ces temps de contraintes budgétaires, les autorités en matière d'archéologie encouragent en priorité la constitution de «réserves» archéologiques, zones névralgiques à protéger dont l'exploitation scientifique et patrimoniale est remise à plus tard.

\section{Quelle place pour le Moyen Âge?}

Actuellement en France, sur les quelque 350 sites de toute nature, réputés visitables en France, les programmes de mise en valeur relevant de la période médiévale, toutes thématiques confondues, représentent moins de $20 \%$ des aménagements. Pourquoi une si faible proportion? On l'a vu, les choix de conservation à l'origine des sites et parcs archéologiques sont étroitement liés à l'histoire de la recherche et des institutions en charge du patrimoine. À cet égard, la «jeunesse » de l'archéologie médiévale en tant que discipline scientifique (développée en France plus d'un siècle après la Préhistoire, par exemple) explique en partie le déficit observé. Mais une autre explication s'impose : encore aujourd'hui, l'architecture médiévale conserve un très fort impact sur le 
paysage bâti de la majorité de nos agglomérations et la frontière entre notre cadre de vie ordinaire et les vestiges archéologiques n'est pas étanche : la nécessité de constituer des « enclaves» se fait moins sentir que pour les vestiges des périodes plus anciennes. Ces conditions conduisent naturellement à envisager d'autres voies pour assurer la conservation $\mathrm{du}$ patrimoine médiéval, notamment par la réglementation sur l'urbanisme, comme les secteurs sauvegardés ou les zones de protection du patrimoine urbain et paysager.

Mais revenons au thème du colloque : dans la catégorie du patrimoine religieux urbain, la nature des présentations et des mises en valeur est loin d'être homogène. La formule des sous-sols ou cryptes archéologiques est la plus répandue, héritière de fouilles anciennes par des architectes et des archéologues en activité entre les deux Guerres mondiales ou dans l'immédiat Après-Guerre. Nombre de réalisations consistent, actuellement, à poursuivre l'étude et la valorisation de ces cryptes (Saint-Laurent de Grenoble, Saint-Jean de Poitiers, Notre-Dame de Paris...). En réalité, il faut attendre la dernière génération d'actions de sauvegarde (années 1970-1980), pour envisager des programmes de conservation et de mise en valeur plus risqués, parce que consacrés à des ensembles du Moyen Âge nouvellement exhumés, on l'a vu, à l'occasion de grandes fouilles de sauvetage dans les centres urbains. Et, précisément, ces programmes visent des vestiges dotés d'une forte teneur identitaire, puisqu'il s'agit, pour la majeure partie, des restes des premiers édifices chrétiens, groupes cathédraux, baptistères... Certes, avec les vestiges de l'Antiquité ils partagent les arguments principaux, ceux de la monumentalité, de l'esthétique architecturale et ornementale. Mais pour les promoteurs de leur étude et de leur conservation, ils sont des témoins historiques manifestes d'une étape particulière de l'histoire du pays, dont la signification doit être transmise non seulement par des écrits, mais dans la pierre. Quel est donc ce message à destination du public ? À vrai dire, un message un peu paradoxal : celui d'une rupture de civilisation: le passage de l'Antiquité païenne à l'ère chrétienne, autrement dit à notre ère ; mais aussi celui d'une forme de continuité chronologique, topographique et stylistique entre le cadre monumental de la ville moderne et les témoignages du passé. Il est d'ailleurs intéressant de noter que, quel que soit le bord des institutions politiques concernées, les pouvoirs publics contribuent alors à la promotion des édifices religieux retrouvés. Il paraît naturel, évident, que la signification de ces lieux qualifiés de "paléochrétiens" transcende tous les clivages. La religion chrétienne y est, de fait, présentée comme la religion de la France, sans que, à l'époque, on se pose la question de la place d'autres religions. On ne soulève pas davantage la question de la justification des investissements des pouvoirs publics laïcs, puisque l'archéologie se place objectivement au service de l'Histoire.

\section{Pour conclure la table ronde}

$19 \mathrm{Au}$ fond, une grande partie des expériences présentées lors de la table ronde de Luxeuil trouve son origine dans le contexte qui vient d'être décrit pour la France et, sans doute, assez proche des circonstances qu'ont connues nos voisins suisses, italiens ou belges. Quelques décennies plus tard, des moyens supérieurs et une meilleure coordination des services en charges du patrimoine ont favorisé leur aboutissement. Mais, à partir des présentations et des discussions qu'elles ont suscitées, on prend conscience que c'est l'inspiration même des réalisations qui s'est renouvelée : les programmes se fondent 
désormais sur une approche archéologique large de l'environnement bâti et cherchent à rétablir la cohérence du tissu urbain ancien. La continuité avec les strates des périodes pré et post-médiévale est recherchée, tout comme le lien topographique et culturel entre domaines religieux et profane.

\section{Questions de vocabulaire}

Pour commencer, revenons sur le titre de la table ronde «Présentation et mise en valeur des sites archéologiques religieux en milieu urbain»; il appelle en effet quelques commentaires, en raison des sous-entendus qu'il comporte et dont la teneur s'est révélée au fil des communications. Présentation et mise en valeur sont évidemment considérées ici en tant qu'aboutissement d'une longue démarche qui débute par l'étude scientifique. Le site archéologique est donc ici compris comme l'ensemble monumental, ruiné ou tout ou partie conservé, révélé par l'archéologie, la fouille proprement dite ou l'analyse du bâti. Le qualificatif religieux, quant à lui, sous-entend qu'il s'agit d'édifices voués au culte chrétien, d'où découle, de fait, que l'on s'intéressera aux constructions postérieures à l'Antiquité. Enfin, la précision du contexte urbain a des implications sur la nature des édifices concernés et le parti scientifique adopté (ensembles cultuels majeurs contribuant à la composition urbaine), mais aussi sur les problématiques architecturales et culturelles de la restitution au public.

21 Aussi encadré soit-il, le thème de la table ronde s'est révélé offrir une grande diversité de cas et d'expériences: expériences anciennes, offrant la possibilité d'un retour sur expérience, ou récentes, voire en cours; réussites ou échecs; vestiges révélés par l'archéologie sédimentaire et/ou l'archéologie du bâti; vestiges authentiques et/ou restitutions ; présentations en sous-sol ou en plein air, etc. La table ronde a également permis l'expression de divers points de vue : celui du scientifique et du conservateur, celui du maître d'œuvre et du restaurateur, celui du maître d'ouvrage et du gestionnaire. De cette diversité, il est possible de faire ressortir quelques faits saillants qui ont déterminé, orienté ou jalonné les réalisations présentées par les communicants.

\section{À l'origine des programmes : la recherche scientifique}

Dans tous les cas exposés, le fait générateur du projet de conservation est bien la reconnaissance de l'intérêt historique des vestiges révélés par l'archéologie. Aux recherches conduites dans le cadre de programmes scientifiques rationnels, dans la durée et par des équipes institutionnelles ancrées sur place (comme le Centre d'études médiévales à Auxerre, le service cantonal à Genève...), s'opposent des recherches conduites dans les conditions plus ou moins difficiles de l'archéologie préventive (baptistères de Grenoble et de Brioude), liées à des programmes d'aménagement urbain. À noter que les fouilles préventives de Brioude ont été complétées par un programme de fouilles triennal. Quelles que soient les conditions de réalisation plus ou moins favorables des fouilles et études, elles sont toutes à l'origine de la désignation de leur objet comme une portion d'un patrimoine commun à sauvegarder. 


\section{Des programmes de longue haleine}

23 La question de la programmation, au sens d'objectifs assignés et de modalités de réalisation, a été évoquée par presque tous les participants. On constate que les exemples d'une démarche régulière, logique et continue sont les plus rares. En effet, toutes ces actions s'inscrivent dans la durée et sont soumises aux aléas de toute entreprise collective. En général, plusieurs décennies se sont écoulées entre l'incitation et la réalisation du programme de mise en valeur (dix ans pour le baptistère de Grenoble, vingt ans pour Saint-Martin d'Angers et Saint-Germain d'Auxerre et trente pour le square des Bénédictins à la Charité-sur-Loire !). Des décennies qui intègrent la recherche archéologique, les prises de décision, l'élaboration des projets de présentation, la réunion des financements, mais aussi les phases de doute, les changements politiques... Mais, dans le meilleur des cas, lorsque l'enquête archéologique a à la fois précédé, accompagné et orienté le programme de mise en valeur, comme à Saint-Germain d'Auxerre et à la cathédrale de Genève, la durée est également appréciée comme un des atouts de la réussite parce qu'elle a permis de préserver le rythme du processus d'analyse. Le témoignage des expériences conduites à Aoste, à l'échelle de la ville, restituant comment des objectifs de conservation et de mise en valeur à long terme, fondés sur une programmation scientifique durable, assumée par des équipes pérennes, fait figure de situation idéale. Mais, généralement, le temps de la recherche n'est pas celui de l'économie et de la politique et cette différence d'appréciation pèse parfois sur le déroulement des opérations.

La plupart des communications ont abordé la question des partenariats qu'impliquent les étapes de la protection et de la mise en valeur et ont décrit le cheminement d'une entreprise qui n'est plus l'affaire des seuls spécialistes. Des expériences négatives (en particulier dans le domaine de la "scénographie») ont été mentionnées, mais la majorité a fait état de collaborations fructueuses au moment de décisions politiques courageuses (La Charité-sur-Loire, Angers, Grenoble, Brioude), parce qu'elles n'allaient pas dans le sens de la satisfaction immédiate des aspirations des administrés. Les communicants ont également montré que la qualité du programme et des résultats est tributaire de celle des échanges entre archéologues, maîtres d'œuvre et maîtres d'ouvrage.

\section{Effets et enjeux du contexte urbain}

En contexte urbain, les vestiges faisant l'objet d'un programme de mise en valeur sont en général en liaison directe, visuelle ou matérielle, avec d'autres édifices, tout ou partie en élévation. Les cas présentés ont montré qu'une partie du projet scientifique consistait à établir, ou à rétablir, le lien intellectuel et physique avec l'environnement bâti. Le cas de Brioude a démontré que le travail sur le patrimoine environnant l'abbatiale s'intégrait à une démarche globale à l'échelle de l'agglomération, notamment par le traitement des voieries et circulations. Dans un tout autre contexte de pression urbaine, à Saint-Denis, l'aménagement des abords de la basilique est davantage affaire d'urbanisme que de démonstration archéologique; dans ce cas, il est sensible que les données archéologiques " échappent » aux spécialistes et sont sources d'inspiration lointaine des aménagements de surface. 
26 La volonté de recréer, à partir de la réhabilitation du square des Bénédictins, une organisation urbaine malmenée depuis la Révolution, a été soulignée dans l'expérience de la Charité-sur-Loire comme une volonté conjointe des archéologues et des maîtres d'œuvre et d'ouvrage. Ainsi, les vestiges monumentaux de Saint-Laurent sont sortis de l'isolement que leur conférait leur statut de ruines, grâce aux points de vue ménagés et aux circulations retrouvées. À Aoste, comme à Genève, on s'attache à ce que les différents sites mis en valeur ne soient pas conçus comme des interventions ponctuelles, mais comme autant d'ouvertures sur une compréhension plus générale de la ville médiévale.

\section{Le message culturel entre authenticité et recomposition}

27 La question du message à l'adresse du public a été posée d'emblée par Christian Sapin en introduction à la table ronde: "Quelle réalité matérielle souhaitons-nous transmettre [à travers les vestiges présentés] ? Quel équilibre avec une réalité virtuelle [issue de l'interprétation et de la restitution] souhaitons-nous conserver?»

La difficile sauvegarde de l'authenticité des vestiges a été souvent évoquée, tant sous l'angle des mesures techniques en assurant la préservation que sous l'angle de leur lisibilité in fine. Ont été notamment signalées, à plusieurs reprises, les contradictions qui pouvaient se faire jour entre la démarche de l'archéologue et celle des concepteurs des travaux de valorisation : la démarche de l'archéologue, aux prises avec le paradoxe qui consiste à conduire une étude par nature destructrice tout en souhaitant conserver la plus grande part de vestiges intelligibles; celle du maître d'œuvre face aux nécessités de la conservation et de l'aménagement, réclamant des interventions sur les vestiges, voire des reconstitutions plus ou moins poussées.

Par comparaison avec les cas de conservation et de mise en valeur facilités par des espaces couverts (cryptes ou sous-sols archéologiques de Grenoble, Genève, Auxerre), les aménagements en plein air posent, évidemment, des problèmes spécifiques. À cet égard, la solution adoptée pour préserver et mettre en valeur les vestiges de l'église Saint-Laurent à la Charité-sur-Loire, au terme de plusieurs décennies de tribulations, montre une solution à la fois efficace et esthétique, qui préserve l'authenticité des vestiges.

Les frottements entre les scientifiques et les scénographes, signalés, entre autres, à Saint-Martin d'Angers, témoignent probablement de questions beaucoup plus importantes que de simples problèmes de forme et pourraient révéler la difficulté qu'il $\mathrm{y}$ a concevoir et à transmettre le message historique et culturel dont les vestiges sont porteurs. Au plan général, autant les acteurs ont su théoriser sur les questions techniques, sur les doctrines de restauration, autant ces mêmes acteurs se sont peu exprimés sur le contenu du message et le public visé. On constate que les expériences les plus probantes sont celles qui combinent deux conditions favorables: lorsque l'élaboration du projet de conservation et de mise en valeur et l'étude scientifique sont en grande partie simultanées, se nourrissant mutuellement; et lorsqu'un chef de projet, mandaté par le maître d'ouvrage, est clairement impliqué dans l'élaboration du message, comme intermédiaire entre les scientifiques et les concepteurs, d'une part, et les futurs "bénéficiaires" de la mise en valeur, d'autre part (collectivité locale et public). Ce fut le cas à Genève (avec une fondation privée) et à Auxerre (avec les 
services de la Ville d'Auxerre), pendant les vingt ou trente années qu'ont duré l'élaboration et la réalisation de ces cryptes archéologiques.

31 Au-delà de la question du pouvoir d'évocation de vestiges dits " authentiques », le sens du message d'ensemble est bien d'offrir une sorte de seconde vie aux témoins d'une époque révolue. Un tel enjeu s'appuie obligatoirement sur une recomposition des rapports du passé au présent, sur une forme de «fabrication » d'une réalité nouvelle.

Au fond, l'exemple du square Castan, conçu au milieu du XIX ${ }^{\mathrm{e}}$ siècle à Besançon, dont la récente restauration a su conserver l'esprit, est une illustration bien plus que folklorique de la fabrication d'un concentré du temps, même si, bien entendu, il serait inconcevable aujourd'hui d'en donner une traduction aussi radicale.

La question posée par les communicants est bien celle de l'ampleur et de la forme de cette recomposition et de sa capacité à atteindre le public dans la durée. De telles ambitions conduisent parfois les concepteurs à sortir des limites du site proprement dit, limites topographiques, mais aussi limites conceptuelles. À Grenoble, Auxerre ou Genève, la mise en valeur des témoins archéologiques s'inscrit dans des programmes immobiliers et muséaux d'ensemble: le propos culturel et ses divers vecteurs incorporent les vestiges dans une sorte « d'offre globale » à l'adresse d'un large public. C'est aussi la solution recherchée à Angers, après l'aboutissement de la mise en valeur de l'église Saint-Martin, qui devrait être l'occasion de redonner un sens aux espaces en partie délaissés de la Maison du tourisme et de l'abbaye Saint-Aubin. À la Charité-surLoire, les vestiges médiévaux deviennent le cadre de nouveaux usages, tels que les festivals. On entre dans le domaine de l'animation.

\section{Assurer la permanence des aménagements et la pérennité des vestiges archéologiques}

33 Tous les cas évoqués montrent la difficulté qu'il y a à maintenir, à terme, l'intérêt et l'engagement des maitres d'ouvrage autant que celui du public. La capacité des équipes gestionnaires à actualiser le message culturel ne va pas de soi et cet ajustement réclame l'implication des acteurs bien au-delà de l'enthousiasme de la réalisation. Par exemple, l'effort considérable consenti par la fondation gestionnaire, avec l'appui des archéologues cantonaux, pour la refonte de l'appareil didactique de la crypte archéologique de la cathédrale de Genève, donne une bonne illustration de ces nouvelles nécessités. Il s'agit d'adapter le discours, sur le fond et dans la forme, à l'évolution des goûts et pratiques sociales et culturelles.

On prend alors conscience que la gestion «courante » des sites, une fois assurée leur étude, leur préservation et leur mise en valeur, ne saurait se passer de la mobilisation régulière des scientifiques. Elle implique une gestion de proximité et une continuité des investissements publics ou privés (collectivités, fondations, associations), conditions difficilement réunies dans les exemples français. On l'a dit plus haut, l'intégration du propos archéologique à une offre culturelle diversifiée contribue à la longévité des aménagements et, donc, à la pérennité des vestiges eux-mêmes. C'est sans doute le défit le plus difficile à relever. 